ORIGINAL ARTICLE

\title{
Perinatal pathology in the context of a clinical trial: attitudes of neonatologists and pathologists
}

\author{
C Snowdon, D R Elbourne, J Garcia
}

Arch Dis Child Fetal Neonatal Ed 2004;89:F204-F207. doi: 10.1136/adc.2003.012732

See end of article for authors' affiliations

......................

Correspondence to:

C Snowdon, Centre for

Family Research,

University of Cambridge,

Free School Lane,

Cambridge CB2 3RF, UK;

cms1000@cam.ac.uk

Accepted 2 October 2003
Objective: To describe the attitudes of neonatologists to trial related perinatal postmortem examinations (PMs), in the light of declining perinatal PM rates and poor levels of participation in pathology studies. Methods: A qualitative study was carried out, using semistructured interviews. Twenty six neonatologists from five UK neonatal units were interviewed; five UK perinatal pathologists also contributed to the study. The professionals involved were all linked to one or both of two neonatal trials.

Results: Pathologists expressed concern over the difficulties experienced in UK perinatal pathology and the impact on research of inadequate levels of samples. The interviews with neonatologists reveal discomfort over approaching bereaved parents for PMs, and a widespread concern that parents should not be further distressed or feel under pressure to consent. Although there was support for neonatal trials, the study highlights a view that PMs may be unnecessary if the cause of death seems apparent or when a baby was born prematurely, and a devaluation of PMs among some younger staff. Poor rates of participation in pathology studies may be accounted for by a notable sense of disconnection between trial interventions and pathology studies.

Conclusions: Neonatologists were concerned to protect vulnerable parents and varied in whether they saw this as compatible with inclusion in trial related pathology studies. Dedicated research is needed to document and gain an understanding of the consent process and should examine the usefulness and impact of consent forms. It should assess whether professionals might benefit from training, to help parents to come to their decisions.

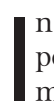
the first of three linked papers ${ }^{1}$ we argued that potentially complex reasons for declining perinatal postmortem (PM) rates warrant further research. This paper reports a qualitative study of views of 26 neonatologists and five pathologists involved in two neonatal randomised controlled trials (RCTs). A further paper ${ }^{2}$ reports interview data from a small number of bereaved parents associated with these trials.

\section{METHODS}

\section{Neonatologists}

The neonatologists were associated with one or both of two RCTs. The INNOVO trial (www.innovo-trial.org.uk) compared giving babies of any gestation either inhaled nitric oxide using a ventilator or standard ventilator care. The CANDA trial ${ }^{3}$ compared two surfactants for preterm babies. The INNOVO trial had a PM protocol and specific organ studies (heart, lungs, and brain). Although the CANDA trial had no specific PM study, if PMs were carried out, information from lung tissue was used to supplement trial findings.

Thirty one neonatologists involved with recruitment to these trials from five centres were approached to participate in the qualitative study during 1999-2001. Centre A recruited neonates to the INNOVO trial only, B-D to both trials, and E to the CANDA trial only. One neonatologist declined to be interviewed. The semistructured interviews covered a wide range of issues raised by RCTS. They were all conducted by CS, tape recorded, fully transcribed, and analysed with the assistance of a computer based qualitative analysis package, Atlas-ti. ${ }^{4}$ CS was primarily responsible for the analysis, but DE and JG also read all transcripts and agreed the analysis.

The subject of PMs was first raised in the fourth interview, and then incorporated into the interview schedule. Data are therefore presented from 26 interviews. Eleven interviewees were consultants. Twenty three were male. Ages ranged from 30 to 54 , mean 37 years. Eight were linked to the INNOVO trial only, eight to the CANDA trial only, and 10 to both trials.

\section{Pathologists}

The pathologists' views were collected after analysis of the neonatologists' views. Six pathologists associated with the INNOVO trial pathology study and one associated with the CANDA trial were invited to respond to the issues raised. Five responded in writing or by telephone. Their anonymised comments contextualise the neonatologists' views.

\section{Ethics}

Relevant multicentre and local research ethics committee approvals were given for the two trials and for this qualitative study.

\section{RESULTS}

\section{Pathologists' views}

Pathologists expressed much concern over the difficulties experienced in UK perinatal pathology. In addition to a long standing problem of few specialist training posts, they felt their profession was under a great deal of strain, "a very sad state indeed".

[Pathologists] have taken a huge beating and are giving up. ...Vital research cannot be done, and it has a huge knock on effect. I used to see 150 baby [organs] per year. Last year I had 13. If I have this level of material I cannot make diagnoses, cannot help parents to understand why

Abbreviations: PM, postmortem examination; $R C T$, randomised controlled trial 
their baby died and will not retain my own diagnostic skills for lack of experience.

Another pathologist had received only one sample for the INNOVO trial in the previous year. A third described sharply declining rates since involvement in an earlier neonatal RCT, and felt this is "to the detriment of clinical care and to the extreme detriment of the parents who fail to get useful information to help them come to terms with their loss."

Pathologists were asked to reflect on the value of pathology studies for neonatal RCTs. Their views were clear, arguing that there is an inherent danger in poorly evaluating potential ill effects of experimental treatments.

[Postmortems] should be almost mandatory in any situation where any kind of therapy is being tested in a clinical trial. ...[We should not] treat these children as some kind of experimental laboratory animal. Far from it. It is the case however that each individual child who receives treatment and each individual child who unfortunately succumbs to their pathology while under treatment represents an irreplaceable source for the assessment of innovative therapies. We have a responsibility to ensure not only efficacy of treatment in a positive sense but also absence of deleterious effects. It would be a great sadness if these treatments were to escape into the general usage and subsequently be identified as being deleterious in the years to come.

As pathologists are not involved in the consent process, they depend on neonatologists as mediators, with the potential to affect the situation positively or negatively. When consent rates were low, they felt this related to professional discomfort and lack of knowledge about the role and value of PMs.

[They] must be seen as a continuation of the trial not as a further intrusion in the harrowing process that parents suffer leading up to the death of their children. It is critical that the consent process is handled by people who are committed to all aspects of the trial protocol and do not have an agenda which excludes the PM from that process. There is rather too much of a view that the child has "suffered enough" which intrudes in the process of asking for a PM examination. It is entirely appropriate that parents should be encouraged in the belief that they are making a significant contribution to the greater good. Many parents find some satisfaction in the hope that their loss can prevent similar things happening to other children.

\section{Neonatologists' views}

\section{Responsibility to the trial and to the parents}

Neonatologists were asked whether a baby's enrolment in a trial affected their approach to parents. Responses were linked to the responsibility they felt to the trial, to parents, and how they viewed the impact of the approach.

They articulated varying degrees of responsibility to contribute to RCT pathology studies, which appeared to be determined by their knowledge of trials and their allegiance to parents. They varied in familiarity with PM processes generally and specifically for the INNOVO and CANDA trials. Most consultants were knowledgeable about requirements and described alternatives such as limited PMs if parents are uncomfortable with certain procedures. ${ }^{5}$ Neonatologists' views are divided into three broad groups: those suggesting a sense of responsibility that was (a) equal, $(b)$ divided, or $(c)$ prioritised.

\section{A sense of equal responsibility}

Neonatologists with a sense of equal responsibility viewed their RCT contributions as important, and felt it is possible to combine these with full consideration of the needs of families. Within this group, some described a moral responsibility to contribute to trials, with a consultant arguing that he feels "mandated" to do so. Another feels "even more of an imperative to try to get PM tissue within the trial context". In his view, not carrying out a PM would be an "opportunity lost". These neonatologists felt they could combine what they saw as their duties to individuals with duties to the wider community.

\section{A sense of divided responsibility}

Some neonatologists described with some anxiety, their feelings of responsibility to research, as well as to families in their care. They exhibited some doubt over whether the two could be served by inclusion in PM studies. For some, there was great tension between the ideal of contributing to research while also providing care.

With the knowledge that trials are used to improve care, one neonatologist described a moral pressure to gain consent for a PM. He foresaw a potential conflict of interests between individuals and the wider community.

There would be some pressure on the person requesting the autopsy, that they do so for the benefit of the trial and prospective future babies who might be enrolled in that trial. ... [lf] it was causing problems and causing deaths then it clearly would be to everyone's advantage to find out that early. You have to balance that against the parents' wishes to not have an autopsy.

The sense of pressure was a concern for a few. Intellectually they felt that PMs are important, and that inclusion in a trial means that there is a responsibility to explore the possible impact of interventions. Practically and emotionally, however, the shift from providing care in a clinical context to a research context could cause great problems.

[Normally] if you say to the parents, "Can we do a postmortem?" and they say no, you say, "Well, OK." Whereas ... you're under a little bit more pressure 'cos you're in a study to actually then push them a little bit harder and say, "Look, we really do need this, this will help other babies." They say, "Look, I've already helped other babies [by being] in the study to start with, now you're asking me to ... chop my baby up, I just want his pain to end." So I do feel ... they've got a point there.

\section{A prioritised sense of responsibility}

Some expressed the view that parental needs should be prioritised, while responsibility to trials was attributed varying degrees of importance. Neonatologists could see trials as important but secondary to parental needs. A consultant commented that consent for a PM for neonatal RCT purposes "wouldn't be top of my priorities." Another consultant who aims to offer trial related PMs was adamant that there be no further discussion once parents indicate their choice: "if they say no, they say no, end of story. Looking after the family comes way before the trial." 
Some neonatologists described PMs only in terms of the patient for whom they had clinical responsibilities and were unaware of their value for neonatal RCTs. They seemed unaware of a role other than to ascertain cause of death and argued that trial enrolment made no difference to consent. Although less senior neonatologists were often responsible for initial trial recruitment, some were unfamiliar with pathology elements of the trial. This may not be surprising given the various career stages of interviewees, and supports similar findings in the literature. ${ }^{6}$ Few of these had considered that trial participation may change the importance of a PM, the grounds on which it is required, or the information that may be requested by parents. It was striking that, in the interviews (which explored various ways in which practice and parental experiences were shaped by inclusion in neonatal RCTs), the PM and the RCT were often considered to be unconnected. This was particularly clear when four neonatologists who had recruited to the INNOVO trial, including a consultant, were unaware of the pathology study. Some neonatologists placed little value on PMs themselves, arguing that they were unnecessary as it is often clear why a baby has died (see below).

\section{PMs solely as trial requirements}

If there is no query over cause of death, but a baby could be included in a neonatal RCT pathology study, essentially newly bereaved parents could be asked to permit a PM for purely altruistic reasons. Some neonatologists saw it as appropriate to request such a PM, albeit carefully, some saw the value but viewed it as inappropriate, and some saw it as unnecessary, particularly for preterm babies where much is known about causes of death. One consultant felt that few doctors would be enthusiastic about approaching parents in such circumstances. This is borne out by low preterm PM rates in the INNOVO trial (26\% preterm $v 67 \%$ term) and the comments of a less senior neonatologist.

[Postmortems] are of very limited value. You usually know why a baby has died. So ... why cut them up. If you don't know why a baby has died then it's perfectly valid ... but if you've got a prem baby ... I think that the parents might well think that you're pushing it because of the trial.

When doctors were uncomfortable and when they felt a PM had little to offer parents, there were clear difficulties. A specialist registrar who was well informed about trial requirements felt it was far more discomforting to ask for a PM for a trial than purely on clinical grounds.

[In] a conventional postmortem you restrict the area to somewhere you're unsure [eg] the cause of death ... [The] problem with ... INNOVO [is] that even in babies who died of something completely different ... or we know the cause of death, their brain and a chunk of their lungs and a chunk of their heart are going to go to different areas of the country, and the baby's going to be buried without those organs ... There are lots of issues around that I do feel a bit uncomfortable with.

\section{Concerns over application of pressure}

Regardless of where doctors saw their responsibilities, they were concerned that bereaved parents should not be pressured to consent to a potentially disturbing procedure. There was also concern that requesting a PM for the benefit of others may be construed as "emotional blackmail" or "a bit callous". There was also concern over the possible inference that a baby may have been harmed as a result of the parental decision to join a trial.

[lt's] almost unfair to suggest to them that there's more of a reason to do a postmortem on their baby than another baby who wasn't part of the trial. [It] ... might suggest that there might be something that the trial did that we need to find out.

Neonatologists commonly said that they back down as soon as they sense parents' discomfort. One neonatologist said that when he realises that parents are going to decline, he does not feel that it is "appropriate in any way to push beyond that." Another felt that dropping the subject very quickly eased his own situation.

I never felt under pressure to get parents to consent to a postmortem, in fact quite the opposite. If the family didn't want [one] we really left it very rapidly.

\section{Management of consent}

If parents are approached, local practice and legal requirements have to be carefully balanced with parental needs. Current Department of Health guidelines state that consent forms should involve decisions about which body parts may be studied (a full or limited PM), how body parts should be disposed of, and whether samples may be used and retained for research purposes. ${ }^{7}$ At the time of the trials, information followed the then standard guidelines and was much less explicit, but by the time of the present study, most centres had drawn up their own very detailed forms. There was some variety in how these forms were viewed and used. They could be seen as a means of bringing parents to an understanding of their options, but could also give professionals a sense of being "covered by the consent form" precisely because they refer to research. They could be used after an initial discussion, with parents being given the forms to read on their own when they felt able to do so. This could be due to the time it takes to go over long, legalistic documents, or to allow parents some privacy. They could also be used to frame discussion of the details of a PM, including the collection of research samples.

Confronting parents with unsettling information and asking them to make decisions, such as whether or not to permit removal and retention of a brain, can be stressful for everyone. Whereas some saw detailed information giving as appropriate, others felt it marked a shift to a more defensive professional position and placed too great a burden on parents. One neonatalogist described it as "absolutely ridiculous" and "not fair on the parents"; another asked "how brutal do you really want to be with bereaved parents?" One senior consultant commented:

We have a consent form that actually talks about removal of the brain ... [There's] no question at all, it becomes uncomfortable. You are trying to support parents at a terrible time ... [but you are also asking] them to do something very horrible to their [baby]... I can see myself refusing post-mortem too.

It is important to note that this consultant had not, however, lost faith in the consent process, which he valued highly. 
On the one hand it's much more uncomfortable for them having to think through that at a time when they're very distressed but on the other hand they're more informed and they've made a clear and informed choice. So it has to be better, I'm in absolutely no doubt at all about that.

\section{DISCUSSION}

The doctors interviewed for this study are working in a difficult climate. There is a worldwide move towards greater openness in research and clinical practice, with a high value placed on the quality of informed consent. Most centres now use detailed forms for consent for both RCTs and RCT related PMs. These two consent processes were, however, often viewed by the doctors in this study as discrete events, not as part of a linear process.

Although some doctors embraced the direction that consent for PMs has taken, openness in discussion of PMs led some to feel that they are engaging in something that is potentially rather "brutal". The need to discuss research further complicates an already sensitive area, and they clearly felt that all parties require some protection in precarious emotional and political circumstances. Most found PM discussions problematic, and all tread a careful path in dealing with bereaved parents. As professionals, they expose themselves to a degree of risk by entering an arena that has caused such a political furore in the United Kingdom, and this can only add to their misgivings.

One response to this situation is to approach parents with a great deal of caution. This can involve immediate discontinuation of discussions when parents are uncomfortable.

Another response is to make selective approaches to those who seem to be coping or when a PM is already indicated for other reasons. The request for samples when a PM is for clinical purposes allows a doctor to put the request in less discomforting terms. Crucially this offers realignment with the role of carer rather than researcher. It may also, however, make the role of samples for trial purposes less clear for some.

A third response which may become increasingly common is to make no approach and to opt out of neonatal RCT pathology studies altogether. A head of department in this study stated that, given the UK political climate, he would be reluctant to ask any parents for a neonatal RCT PM and doubted whether any colleagues would do so.

This undoubtedly offers individual parents protection. However, when few samples are sent for pathology studies, not only are there fewer data on which a trial data monitoring committee can base its recommendations about continuation or otherwise of the trial, but also the scientific rigour of the pathology study is undermined. This is especially the case if samples are sent from a highly selected group. The effects of low numbers of samples are already being felt in the trials world. In anticipation of poor rates, there is likely to be a shift towards simply not including pathology studies in RCTs.

\section{Conclusions}

There are two separate impulses at work here: to tell and not to tell, both based on the desire to protect parents and to ease a professional situation. This reflects an uneasy climate in which the tensions between the expectations of the trials community and the everyday practicalities of caring for families have not always been fully worked through. If professionals are uncomfortable, it is likely that parents will also be. It is therefore important that those involved in neonatal RCTs find a clearer way through the situation which is feasible for clinicians and not to the detriment of vulnerable parents.

To date this is the only study reporting on attitudes to RCT related PMs. There are, however, limitations to this study. The fact that views on the subject of PMs were collected as an adjunct to the larger topic of the professional experiences of recruitment to RCTs means that the study was not set up primarily to explore these issues. It is most important that the concerns doctors and parents should receive further attention, through discussion by the wider research community and through dedicated research. Research should document the consent process and clarify the effect of the difficulties described here. It should assess the steps taken to protect these parties from problems over consent, and most specifically should examine the usefulness and impact of particular consent forms. It should assess whether professionals might benefit from training in the skills that go along with helping parents to come to their decisions. Quite clearly, all parties require a degree of support. Research is needed to decide what that support ought to be.

\section{ACKNOWLEDGEMENTS}

The Nuffield Foundation funded this study. JG worked on this study at the National Perinatal Epidemiology Unit where she was funded by the Department of Health. CS was accommodated by CFR and assisted by Sally Roberts. Nina Hallowell, John Allison, and David Field (and other members of the Project's advisory group) commented on earlier drafts of the paper. We thank the neonatologists and pathologists who gave generously of their time.

\section{Authors' affiliations}

C Snowdon, D R Elbourne, J Garcia, Medical Statistics Unit, London School of Hygiene and Tropical Medicine, University of London, Keppel Street, London WCIE 7HT, UK

C Snowdon, Centre for Family Research, University of Cambridge, Free School Lane, Cambridge CB2 3RF, UK

\section{REFERENCES}

1 Snowdon C, Elbourne DR, Garcia J. Perinatal pathology in the context of a clinical trial: a review of the literature. Arch Dis Child Fetal Neonatal Ed 2004;89:F200-3.

2 Snowdon C, Elbourne DR, Garcia J. Perinatal pathology in the context of a clinical trial: attitudes of bereaved parents. Arch Dis Child Fetal Neonatal Ed 2004;89:F208-11.

3 Ainsworth SB, Beresford MW, Milligan DWA, et al. Pumactant and poractant alfa for treatment of respiratory distress syndrome in neonates born at 2529 weeks' gestation: a randomised trial. Lancet 2000;355:1387-92.

4 Muhr T. A prototype for the support of text interpretation. In: Tesch R, ed. Qualitative sociology. New York: Human Science Press, 1991.

5 Raffles A, Ropel C. Non-invasive investigations are also helpful if permission for a necropsy is refused. BMJ 1995 April 1;310:870.

6 VanMarter LJ, Taylor F, Epstein MF. Parental and physician-related determinants of consent for neonatal autopsy. Am J Dis Child 1987; 141:149-53.

7 Department of Health Clinical Ethics and Human Tissue Branch. Families and post mortems: a code of practice. London: DoH, 2003. 